\title{
What influences our decision to vaccinate? The social amplification of risk framework and vaccination
}

\author{
Laura B. Carper \\ Louisiana State University \\ Baton Rouge, LA USA
}

\begin{abstract}
The current study applies the social amplification risk framework to the anti-vaccination movement, specifically to the social factors that influence the likelihood to vaccinate. A total of 264 participants were recruited using Amazon's Mechanical Turk engine and students from a large southern university. Participants responded to questions about their personal, friend, and family experiences with vaccines, their discussion about vaccines, and trust in vaccine literature (CDC, Facebook, family, etc.). Lastly, participants responded to a modified Duke's social support scale. Results indicated that the likelihood to vaccinate is impacted by several social factors and that those factors can be amplified based on the experiences of others. The results support using the social amplification of risk on individual perceptions of risk.
\end{abstract}

Keywords - Social Amplification of Risk Framework, Anti-Vaccination, Pro-Vaccination, Social Ties, Trust

SUGGESTED CITATION: Carper, L. B. (2019). What influences our decision to vaccinate? The social amplification of risk framework. Proceedings of the International Crisis and Risk Communication Conference, Volume 2 (pp. 12-14). Orlando, FL: Nicholson School of Communication and Media. https://doi.org/10.30658/icrcc.2019.3

\section{INTRODUCTION}

In 1998, Andrew Wakefield and 12 colleagues published a study in Lancet claiming that the measles, mumps, and rubella vaccine (MMR) led to autism in 12 participants. Soon after, many studies attempted to find the same link between MMR and autism; however, none could be found. Eventually, Dr. Wakefield was charged with fraud and had his medical license revoked [1]. However, the damage was done; his anti-vaccination sentiments spread through the use of social media groups such as 'stop mandatory evacuation now' as well as by word of mouth. While vaccination rates in the United States remain high, however, the CDC cautions that certain enclave 'anti vaccination groups' do choose to not vaccinate their children and, thus, create a serious public health concern [2]. Even though a small percentage of parents choose to not vaccinate their children at all, and arguably may never change that decision, what could influence the decision to vaccinate in general?

Studies in vaccination literature have discussed the role of social media [3], power of the personal narrative [4], and patient-provider communication [5]. However, none of these studies included research in the social amplification of risk or the importance of social networks and the likelihood to vaccinate. Considering the impact of narrative, the current analysis seeks to apply the social amplification risk framework to the likelihood to vaccinate by looking at the sources of amplification, the channels, and the social stations of communication in dyadic communication.

\section{LITERATURE REVIEW}

SOCIAL AMPLIFICATION OF RISK FRAMEWORK (SARF)

Kasperson, Renn, Slovic, Brown, Emel, Goble, et al. [6] suggested the social amplification of risk framework (SARF) as a way to systematically link the technical assessment of risk and psychological, sociological, and cultural factors that influence risk perception and risk behavior, specifically that the perception of risk interacts with one's psychological, sociological, and cultural factors possibly amplifying or attenuating public responses to an event.

In the years since the initial conception of SARF, primary research has analyzed the impact of press releases and coverage, risk signal in print media, organizational amplification, and stigma, trust, and imagery implications. Few, studies have analyzed SARF in terms of individual levels of social interaction, the importance of social ties, and social media 
platforms and disseminating risk information, particularly, vaccination.

\section{VACCINE RESEARCH AND SOCIAL SUPPORT}

Research in specific sources of vaccine information (personal and friends), specifically in terms of reporting side effects to immediate members of one's social networks, is limited; research in SARF indicates the importance of personal experiences and friend interactions. Pidgeon, Kasperson, and Slovic [7] suggest that the experience of risk is not only through physical harm, but the result of group and individual interpretations of that risk.

H1. Past negative personal experiences will negatively impact the likelihood to vaccinate.

While personal experiences may play an initial role in deciding to vaccinate, what about family, friends, doctors, and social media as a channel to discuss vaccines? Studies have shown the importance of social support and networks as encouraging the likelihood to vaccinate [8]. Specifically, Ernsting, Knoll, Scheider, \& Schwazer [9] found that the amount of social support was a useful predictor of the likelihood to vaccinate.

H2.1 Past negative experiences with vaccines from family will negatively impact the likelihood to vaccinate.

H2.2 Past negative experiences with vaccines from friends will negatively impact the likelihood to vaccinate.

In addition to past personal and friends' experiences, research in the doctor-patient relationship and vaccines will be explored. Shay, Baldwin, Betts, Marks, Higashi, Street, et al. [10] found that providers who engage hesitant parents about their concerns led to same-day vaccination.

H3.1 Vaccine information from doctors will positively impact the likelihood to vaccinate.

Finally, a primary element of many anti-vaccination narratives is the impact of trust of the government; specifically, the CDC will be measured.

H3.2 If information from the CDC is seen as trustworthy, the participant will be more likely to vaccinate.

\section{METHODS}

\section{PARTICIPANTS}

A total of 264 participants were recruited using Amazon's Mechanical Turk $(n=150)$ and students from Louisiana State University ( $\mathrm{n}=114)$. Of the 264 participants, 81 were male, 179 were female, and 4 chose to not respond. The mean age of the sample was 32.11 with a standard deviation of 12.76. In regards to vaccine information, 224 reported being a pro-vaxxer $(85 \%)$ and 40 reported being an anti-vaxxer (15\%); 207 reported receiving a vaccine, whereas 25 did not. Lastly, 204 said they would vaccinate a child, but 28 said they would not.

\section{VARIABLES}

Participants were asked if they were $1=$ anti-vaxxer (does not support vaccination) and $0=$ pro-vaxxer (supports vaccination). To determine the source of the message, participants were asked questions about personal experiences: (Have you ever had a vaccine? Have you ever had a side effect? Were you told of the possibility of a side effect?), family sources (Do you know of someone in your family who has had a side effect?), and friend sources (Do you know of any friends who have had a side effect?). Finally, questions about trustworthiness of the channels of vaccine information using Likert scale questions with 5=being the most trustworthy and 1=the least trustworthy of the CDC were also asked.

The primary dependent variable was measured as "Would you ever receive a vaccine yourself, and would you ever vaccinate a child?" Both responses are coded as yes $=1$ and no $=0$.

\section{RESULTS}

Logistic regressions were used to analyse the data. The likelihood to vaccinate was negatively influenced by identifying as an anti-vaxxer. Specifically, a chi square (108.51) was performed analyzing the likelihood to vaccinate and being a pro- vs. anti-vaxxer and was statistically significant $(p=0.000)$ with a Cramer's V of -0.68 indicating a negative strong relationship. Two models were used: The likelihood to vaccinate in the future and the likelihood to be an anti-vaxxer. Both models included past personal history with vaccines, family history with vaccines, friends history with vaccines, CDC trust, black only, doctor primary information, gender, and age. The model for vaccinating in the future was statistically significant $\left(\chi^{2}=84.99, p=0.000\right.$, McFadden's pseudo $\mathrm{r}^{2}=0.58, \mathrm{PRE}=48 \%, \%$ predicted correctly $\left.95 \%\right)$, and the model for predicting anti-vaxxer was statistically significant as well $\left(\chi^{2}=137.93, p=0.000\right.$, McFadden's pseudo $r^{2}=0.64, \mathrm{PRE}=60.5 \%, \%$ predicted correctly $94 \%$ ).

Hypothesis 1 was supported. Personally receiving a vaccine in the past $(b=6.23, z=3.81)$ increased the probability of receiving a vaccine in the future from $13 \%$ to $98 \%$. Additionally, the likelihood of being an anti-vaxxer was also analyzed. Those that had a vaccine in the past had a $3 \%$ probability of being an anti-vaxxer compared to 55\% to those who were not vaccinated.

While Hypotheses 2.1 and 2.2 were not supported in the model for vaccianting in the future, friends who had side effects impacted the likelihood to be an anti-vaxxer slightly $(b=1.83, z=2.01)$. Participants who had a friend with a side effect had an $11 \%$ probability of being an anti-vaxxer compared to those who did not with a $2 \%$.

Next, Hypothesis 3.1 was supported for both the likelihood to receive a vaccine as well as being an anti-vaxxer. If the participants' primary source of vaccine information came from a doctor, they had $99 \%$ of vaccinating in the future compared 
to $96 \%$ whose primary source was not a doctor $(b=1.47, z=1.81)$ and compared to $9 \%$ of being an anti-vaxxer for those who a doctor was not their primary source to $1 \%$ for those who the doctor was the primary source $(b=-1.37, z=2.13)$. Interestingly, more research is needed to understand the different sources of vaccine information considering $96 \%$ is still very high.

Lastly, Hypothesis 3.2 was supported for both the likelihood to vaccinate in the future as well as being an anti-vaxxer. Those who reported the highest trust in the CDC had a $98 \%$ probability of vaccinating compared to $7 \%$ for the lowest trust in the CDC $(b=1.61, z=4.23)$. As expected, the highest trust in the CDC resulted in a 4\% probability of being an anti-vaxxer compared to $99 \%$ for the lowest level of trust $(b=-2.67, z=-5.61)$. Clearly, trust has a very important implication on vaccinating in the future.

I have provided a model that has included all significant factors in the original SARF format.

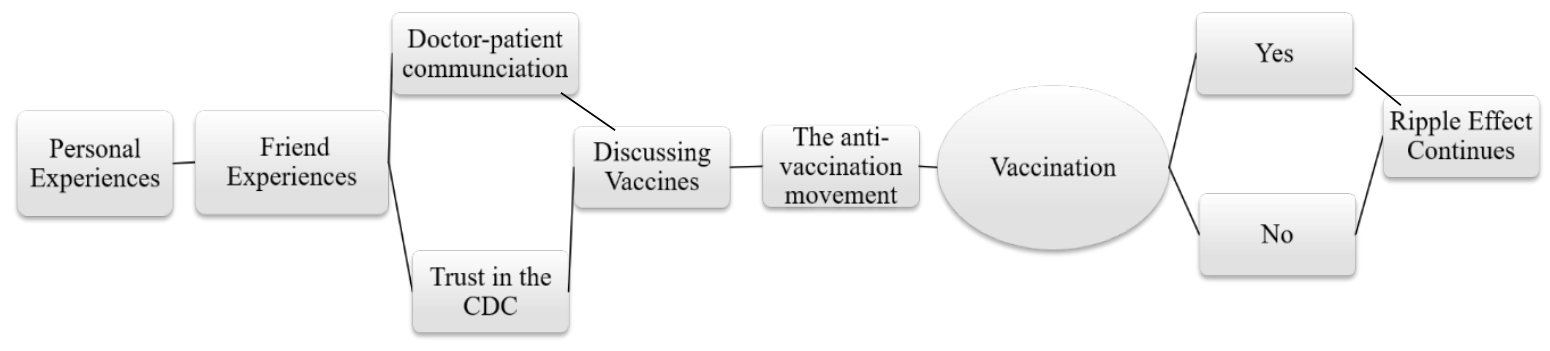

\section{DISSCUSSION}

The current study adds to the breadth of research in SARF by applying the framework to individuals and the personal decision to vaccinate. Results indicate that experiences (whether positive or negative) of individuals, family, and friends can impact the likelihood to vaccinate. Furthermore, individuals were found to respond positively to vaccine information from their doctors. Specifically, doctors were the most common source of information about vaccines, and if participants reported a doctor was their primary source of vaccine information, they were more likely to vaccinate. In addition to doctors, participants who trusted the CDC were also more likely to receive a vaccine than those who did not. Individuals are clearly influenced by opinion leaders, in this case doctors who share the vaccine information of both benefits and possible side effects. Once receiving these messages, individuals then decode and rely on heuristics to determine the ultimate course of action to vaccinate or not.

The decision to vaccinate now is impacted by the anti-vacicnation movement, a form of social protest. The likelihood to be an anti-vaxxer was impacted by similar facets as it was to the likelihood to vaccinate. While I know these two elements are intertwined because anti-vaxxers choose not to vaccinate, I propose to highlight the importance of the vaccination movement as a social process amplified by personal discussion, experiences, government entities such as the CDC, type of information discussed, etc. Ultimately, when individuals choose to not vaccinate, they risk not only getting ill themselves but also impacting their children (anti-vaxxers had a -3.83 decrease in logged odds in vaccinating their children, $p=0.000$. Cramer's $\mathrm{V}=-\mathbf{0 . 6 3}$ ), and the other children those children come in contact with, creating a ripple effect that has large societal implications. Of all of the modes of amplification that need further research, doctor interaction and vaccine are needed. Manca [4] stated, "When these narratives did not fully resolve medical uncertainties, health professionals often explained that either vaccination was beyond their professional responsibilities or that their uncertainties were irrelevant" (p. 114), further highlighting the need for further research. There is a need for medical professionals to understand the breadth of research in their involvement in the likelihood to vaccinate as well as other social factors that the researcher has found that impacts the likelihood to vaccinate.

\section{CONCLUSION}

Though the study had some limitations, there is a clear need for further research into the social stations that influence the likelihood to vaccinate. The current research has argued that the vaccination movement can be analyzed by incorporating SARF to evaluate the messages and message functions and the ultimate goal of vaccination. The current study has found numerous important findings that individuals have reported which increase the likelihood to vaccinate from personal, family, and friends' experiences to discussing the benefits of vaccines, trusting the CDC, and having a doctor as a primary source of vaccine information. By using these results and results of other researchers, messages could be crafted and aimed specifically at anti-vaxxers or those individuals who are unsure. If more research is not continued, the "ripple effect" could encourage further decrease in vaccination rates and increase in preventable diseases. 


\section{Author Biography}

Laura B. Carper (1carpe6@1su.edu) is currently a PhD. student at Louisiana State University; she earned her M.A. in Organizational Communication at Southeastern Louisiana University.

\section{REFERENCES}

[1] Rao, S. \& Andrade, C. (2011). The MMR vaccine and autism: Sensation, refutation, retraction, and fraud. Indian Journal of Psychiatry, 53, 95-96. https://doi.org/10.4103/0019-5545.82529

[2] Kahn, D. (2017). Protecting or polluting the science of communication environment? The case of childhood vaccines. In K. Jamieson, D. Kahan, \& D. Scheufele (Eds.). The Oxford Handbook of the Science of Science Communication (pp. 421-432). Oxford UK: Oxford University Press.

[3] Moran, M., Lucas, M., Everhart, K., Morgan, A., \& Prickett, E. (2016). What makes anti-vaccine websites persuasive? A content analysis of techniques used by anti-vaccine websites to engender anti-vaccine sentiment. Journal of Communication in Healthcare, 9, 151-163. https://doi.org/10.1080/17538068.2016.1235531

[4] Manca, T. (2016). Health professionals and the vaccine narrative: 'The power of the personal story' and the management of medical uncertainty. Health, Risk, \& Society, 18(3-4), 114-136. https://doi.org/10.1080/13698575.2016.1190319

[5] Dempsey, A. \& O'Leary, S. (2017). Human papillomavirus vaccination: Narrative review of studies on how providers' vaccine communication affects attitudes and uptake. Academic Pediatrics, 18(26), S23-S27. https://doi.org/10.1016/j.acap.2017.09.001

[6] Kasperson, R., Renn, O., Slovic, P., Brown, H., Emel, J., Goble, R., . . . \& Ratick, S. (1988). The social amplification of risk: a conceptual framework. Society for Risk Analysis, 8, 177-187. https://doi.org/10.1111/j.1539-6924.1988.tb01168.x

[7] Pidgeon, N., Kasperson, R., \& Slovic, P. (Eds.). (2004). The Social Amplification of Risk. Cambridge, UK: Cambridge University Press.

[8] Opel, D. \& Marcuse, E. (2013). Window or mirror: Social networks' role in immunization decision. Pediatrics, 131, https://doi.org/10.1542/peds.2013-0531

[9] Ernsting, A., Knoll, N., Schneider, M., \& Schwarzer, R. (2015). The enabling effect of social support on vaccination uptake via self-efficacy and planning. Psychology, Health, and Medicine, 20, 239-246. https://doi.org/10.1080/13548506.2014.92095

[10] Shay, L., Baldwin, A., Betts, A., Marks, E., Higashi, R., Street, R., .. Tiro, J. (2018). Parent-provider communication of HPV vaccine hesitancy. Pediatrics, 141, 1-10. https://doi.org/10.1542/peds.2017-2312 